\title{
METHODOLOGY FOR COST BENEFIT ANALYSIS OF SMART GRID USED IN DECISION SUPPORT
}

\author{
QIAN YANG ${ }^{1}$, LOI LEI LAI, CHUN SING LAI2 \\ 1State Grid Energy Research Institute, Beijing, China \\ 2 On leave from Brunel University, UK \\ E-MAIL: yangqian@sgeri.sgcc.com.cn, lailaili@sgeri.sgcc.com.cn
}

\begin{abstract}
:
This paper studies the cost benefit analysis (CBA) methods that can improve the decision support capability of smart grid deployment. Critical review based on various methodologies adopted worldwide has been carried out and detailed analysis was conducted. The finding demonstrates as there are no agreed guidelines for CBA. Also in real-life situation, due to large amount of data and data quality could be low, it is suggested developing CBA methodology based upon intelligent techniques such as neural and evolutionary computing may have a good potential for the future.
\end{abstract}

Keywords:

Cost-benefit Analysis; Smart Grid; Decision Support

\section{Introduction}

Decisions on the formulation and possible implementation of policy regulation depend on appropriate information on the possible effects of decisions regarding cost, benefits, impacts and similar indicators [1][2][3]. As core strategy to improve the efficiency, reliability, economics, and sustainability of the production and distribution of electricity [4], the initiatives of smart grid are taking all over the world. To reduce uncertainties investments of smart grid, it is necessary for utilities and regulators to have a common framework for cost-benefit analysis that properly accounts for the societal benefits that arise from utility investments in reliability [5].

This paper intends to study the cost-benefit analysis methods that can improve the decision support capability of the implantation of smart grid. Section 2 covers issues related to the approach to analysis the benefits of smart grid. Section 3 describes the step of cost-benefit analysis in smart grid project. Section 4 introduces cost-benefit analysis of smart grid based on scenario. Finally, a brief summary is made in the conclusion.

\section{Approach to analysis benefits of smart grid}

Some studies focused on the approach to analysis the benefit of smart grid.

Benefit is an impact (of a Smart Grid project) that has value to a firm, a household, or society in general, and it is not simply a project's performance or intermediate outcomes of the project. [6]. The Electrical Power Research Institute (EPRI) [6] proposes a comprehensive framework for estimating smart grid benefit. The framework identifies the basic categories of benefits, the beneficiaries of these benefits, and the Smart Grid functionalities that lead to different benefits and proposes ways to estimate these benefits, including their monetization. The basic groups of beneficiaries cover Utilities (Utilities are the suppliers of power and include electric utilities that generate power as well as the transmission and the load serving entities that deliver it, customers (customers are the end-users or consumers of electricity), and society (society in general is the recipient of externalities of the Smart Grid). The benefits include four fundamental categories: economic (reduced costs, or increased production at the same cost, that result from improved utility system efficiency and asset utilization), reliability and power quality (reduction in interruptions and power quality events), environmental (reduced impacts of climate change and effects on human health and ecosystems due to pollution), security and safety (improved energy security; increased cyber security; and reductions in injuries, loss of life and property damage). It is the first of its kind to develop a systematic way of defining and estimating the benefits of the Smart Grid, but this methodology has not yet been tested with a real case study [7].

The report [8] of US department of energy presents the benefits of the smart grid from the perspective of each beneficiary in six key value areas and described how the smart grid generates these benefits. The six key value areas include reliability, economics, efficiency, environmental, 
security, and safety, and the stakeholders are delivery company, electricity supplier, residential consumer, and society. The following technology solutions are generally considered when a smart grid implementation plan is developed: advanced metering infrastructure (AMI), customer side systems (CS), demand response (DR), distribution management system/distribution automation (DMS), transmission enhancement applications (TA), asset/system optimization (AO), distributed energy resources (DER), information and communications integration (ICT), and the deployment of these technology solutions is expected to create improvements in the six key value areas. Figure 1 identifies the relationships among these technology solutions and the key value areas. This "many-to-many" relationship illustrates the synergy of smart grid solutions, an affect that must be considered when the scope of the smart grid is planned to ensure the benefits are optimized.

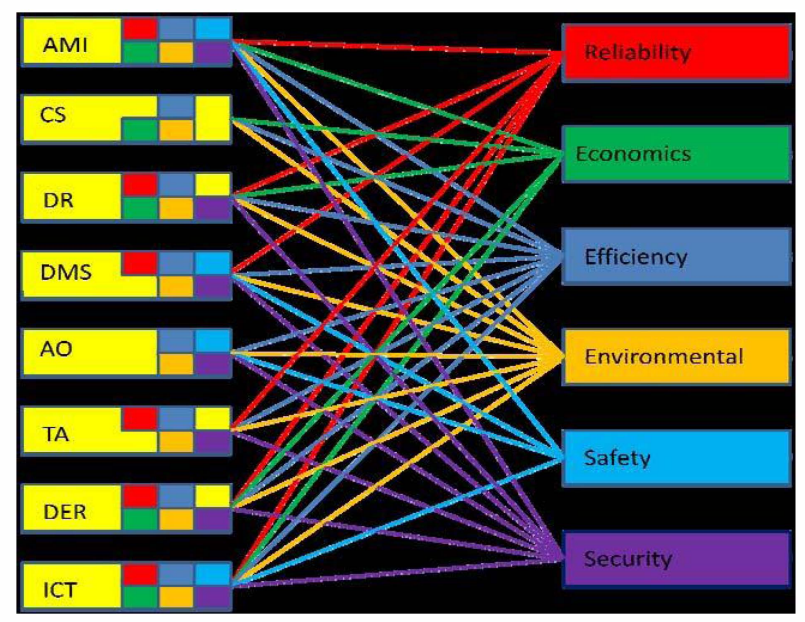

Figure 1: Technology Solution — Key Value Area Relationships [8]

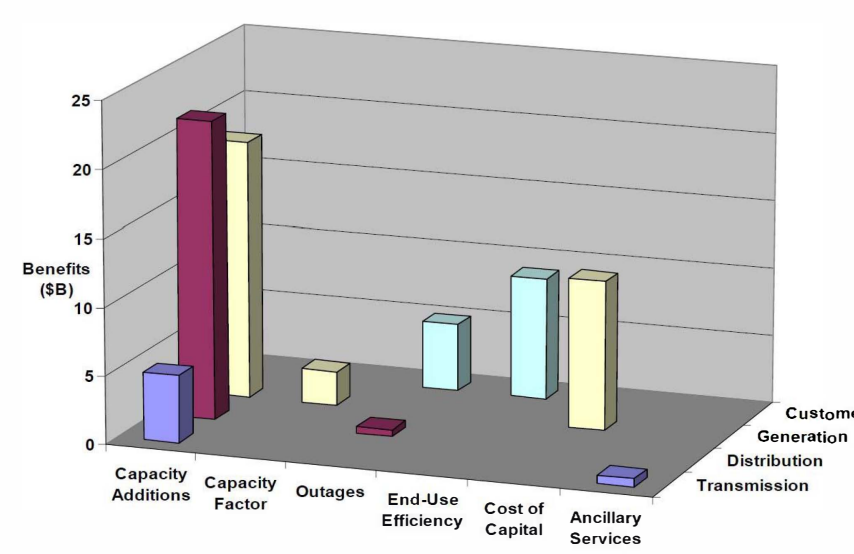

Figure 2. Conservative estimates of the sources of GridWise benefits by utility sector [9]
The report [9] of Pacific Northwest National Laboratory for the U.S. Department of Energy presents a preliminary scoping assessment conducted to envision the general magnitude of several selected benefits the GridWise concept could offer when applied nationally. These benefits accrue in the generation, transmission and distribution components of the power grid, as well as in the customer sector, as indicated in Figure 1. Power generation would benefit from implementing GridWise technologies in several ways including increased revenues, lower investment and operating expenses, and lower capital risk and uncertainty. Although GridWise technologies can offer a number of benefits to the T\&D system (e.g. increased capacity factor, increased market transactions, increased asset utilization and productive asset life, fewer outages and greater revenue growth), only the reduced outage benefit is evaluated in this study. The GridWise vision converts end-use load from a passive element into an interactive system control and feedback mechanism, and this leads to a variety of customer benefits derived from a combination of increased revenues, decreased costs, and reduced risks from uncertainty.

Reference [10] presents a preliminary scoping assessment conducted to envision the general magnitude of several selected benefits the Smart Grid concept could offer when applied in Korea. These benefits accrue in the generation, transmission, and distribution components of the power grid, as well as in the customer sector.

Michael and Josh [5] study the possibility to systematically assess the economic benefits that can result from reliability improvements. They propose that reliability can be measured in a variety of ways. For the purposes of transmission and distribution planning, it is measured in two ways. One approach is to estimate the quantity of unserved energy (in kilowatt-hours) that results, or is expected to result, from service interruptions. This method rests on a very difficult estimation problem - namely, the requirement to estimate the quantity of electricity that would have been demanded if unreliability had not occurred. In turn, objective statistical indicators of reliability are more commonly used when assessing the reliability of systems. Most utilities and regulatory bodies in the U.S. commonly describe the reliability of transmission and distribution circuits in terms of simple, readily obtainable and transparent indicators. These indicators are: SAIDI, SAIFI, CAIDI, MAIFI.

Nenad and Vlado et al. [11] investigated and evaluated the benefit of smart grid solution (SGS) and distribution management system (DMS) (e.g. reduction of power losses, reduction of network outage costs and network development costs, improvement of power quality). At the same time, necessary investment in the installation and application of SGS/DMS is considered.

U.S. department of energy also attempts to quantify the 
additional energy and $\mathrm{CO} 2$ emission benefits inherent in the smart grid's potential contribution to the goal of mitigating climate change by reducing the carbon footprint of the electric power system [12].

\section{The step of CBA of smart grid}

The work of EPRI [6] is then extended to a 24-step framework [13] for performing a CBA. The twenty-four step is as following:

Step 1- Document project information at a high-level

Step 2- Provide a general description of the project purpose including

Step 3- Provide a high-level project summary

Step 4- Provide a high-level project timeline

Step 5- Define the research problem or opportunity that prompted the project

Step 6- Describe any relevant programs or policies that are pertinent to the project

Step 7- Describe the technologies to be deployed and their associated functions

Step 8- Estimate project impacts

Step 9- Describe the baseline(s) required to measure performance

Step 10- Describe the formal hypotheses to be tested in experiments

Step 11- Identify experiments, describe how each will be conducted.

Step 12- Develop a detailed project timeline

Step 13-Define the physical measurements to be take in experiments

Step 14- Define the time intervals for measurements

Step 15-Describe external factors that will require normalization of the data sets

Step 16- Identify data collection points and describe data collection instructions

Step 17- Identify data testing, screening, storage and retrieval protocol

Step 18- Calculate physical impact measurements by beneficiary group

-Step 19- Convert physical impact measurements to monetary values

Step 20- Identify Smart Grid functions with benefits for multiple beneficiaries

Step 21 - Estimate costs incurred by customers per year for baseline and project

Step 22- Estimate utility costs by function/classification for baseline and project

Step 23- Summarize costs and benefits

Step 24- Communicate results

Reference [7] studies a step-by-step assessment framework based on the work [6] performed by EPRI to identify and assess (in physical terms or through a qualitative description) project impacts and externalities to fit the European context, and proposes a 7-step CBA framework, as indicated in Figure 3. The first step is to provide a summary of the project. Smart grid assets provide different types of functionalities than enable benefits, and the step 2 is mapping assets on to functionalities. Step 3 is to link the functionalities identified in step 2 to the (potential) benefits they provide. The step 4 establishes the project baseline to formally define the 'control state' that reflects the system condition which would have occurred had the project not taken place. Step 5 will quantify and monetize the benefits, and the quantification of cost is in step 6. Finally, the cost and benefit will be compared in order to evaluate the cost-benefit of the project.

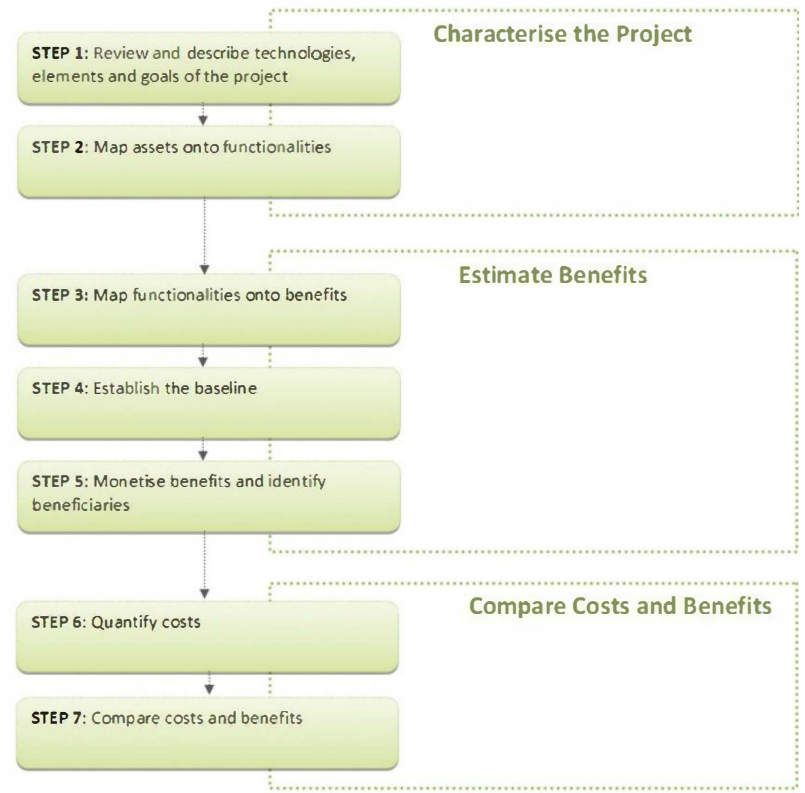

Figure 3. Cost-benefit analysis framework [7]

\section{CBA of smart grid based on scenario}

Some group of studies put forward different scenarios and assumption for the estimation of various smart grid benefits.

Reference [14] presents how AMI meters could provide benefits for customers through time-based rates and to provide additional information. The costs are evaluated in three major categories (capital, O\&M, customer engagement), and benefits are quantified across six major categories (O\&M benefits, capital avoidance, peak reduction, energy reduction, reliability, environmental).The cost and benefit analysis uses 
the NPV methodology by calculating the costs of deploying and maintaining an AMI infrastructure and comparing those costs against the benefits accrued over the twenty-year meter life under base case, worst case and the best-case scenario.

Reference [15] studies the cost-benefit analysis of three scenarios (base case scenario, advanced functionality scenario, multi-metering scenario) of the roll-out of smart metering system. The scenarios of the smart metering roll-out are established based on six general parameters (market model, functionality of smart meters, communication technologies, applicable pricing models, scope of the smart metering roll-out, and speed of the smart metering roll-out). The cost-benefit analysis under each and every scenario is carried out to all users collectively, singling out the costs and benefits to separate user groups. User groups are household urban users, household rural users, commercial users with under $30 \mathrm{~kW}$ of permissible power limit, commercial users with over $30 \mathrm{~kW}$ of permissible power limit, all household consumers, and all commercial consumers.

Reference [16] reports results on real case study on power distribution systems with and without the deployment of smart grid technology. Cost benefit analysis on the use of electric vehicle and customer response was included. It shows that under various scenarios that there is a good impact on the society, environment and security of power supply.

\section{Conclusion}

In this paper, it is clear that there is no scientific proof to confirm which method is better. It seems that each approach is problem oriented. It would be very difficulty if not impossible to develop guidelines to be accepted. Naturally, the outcome of CBA will affect decision support significantly therefore the methodology used needs to be selected with great care.

Although there are many studies touched upon the cost benefit analysis of smart grids, hardly any real case studies are available. Always data quality and 'big' data will be an important issue, the present study suggested developing an intelligent CBA approach to consider data quality and feature extraction in the near future.

\section{References}

[1] Bartlett, Robert V, Policy through impact assessment: institutionalized analysis as a policy strategy, Greenwood Press, New York, 1989.

Centre, State Grid Energy Research Institute, Beijing, China, April 2013. (In Chinese).
[2] Da Ruan, Frank Hardeman and Klaas van der Meer, Intelligent decision and policy making support systems, Springer, Berlin, 2008.

[3] M. Fritz and G. Schiefer, "A multi-level cost benefit approach for regulatory decision support in food safety and quality assurance scenarios", The European Journal of Social Sciences, Vol. 21, No. 2, pp. 177-182, June 2008.

[4] U.S. Department of Energy, Smart Grid / Department of Energy, http://energy.gov/oe/technology-development/smart-grid , Retrieved 2012-06-1.

[5] Michael Sullivan and Josh Schellenberg, "Smart grid economics: The cost-benefit analysis", Renew Grid, April 2011.

[6] Matt Wakefield, Methodological approach for estimating the benefits and costs of smart grid demonstration projects, U.S. Department of Energy, Electric Power Research Institute, January 2010.

[7] Vincenzo Giordano, Ijeoma Onyeji, Gianluca Fulli, Manuel Sanchez Jimenez and Constantina Filiou, Guidelines for conducting a cost-benefit analysis of smart grid projects, EUR 25246, Publications Office of the European Union, 2012.

[8] Understanding the benefits of the smart grid. June 18, 2010. U.S. Department of Energy.

[9] L. D. Kannberg, M. C. Kintner-Meyer, D. P. Chassin, R. G. Pratt, J. G. DeSteese, L. A. Schienbein, S. G. Hauser and W. M. Warwick, GridWise TM: The benefits of a transformed energy system, September 2004.

[10] Jaeseung Song, Hongkeun Jang, Sangbum Yoon, and Byunghoon Min, "Smart grid: The benefits of a transformed energy system in Korea", Seoul National University, August 2009.

[11] Nenad Katic, Vlado Marijanovic and Izabela Stefani, "Smart grid solutions in distribution networks cost/benefit analysis", 2010 China International Conference on Electricity Distribution (CICED), China, IEEE, pp.1-6, September, 2010.

[12] Pacific Northwest National Laboratory, The smart grid: an estimation of the energy and CO2 benefits, U.S. Department of Energy.

[13] M. Wakefield, Guidebook for cost/benefit analysis of smart grid, EPRI, December 2012.

[14] CL\&P AMI and dynamic pricing deployment cost benefit analysis, Docket No. 05-10-03RE01, Department of Public Utility Control, 2007.

[15] Cost-benefit analysis of the roll-out of smart electricity metering grid in Lithuania, Report, Ernst \& Young, Sept 2012.

[16] Analysis on smart distribution grid operation and the research \& development of the decision support system and its applications, Internal Report, Energy Strategy, Planning, Policy Support, Research and Development 